\title{
EuGl: a novel resource for studying genomic islands to facilitate horizontal gene transfer detection in eukaryotes
}

\author{
Frederick Johannes Clasen ${ }^{1,2^{*}}$, Rian Ewald Pierneef ${ }^{1}$, Bernard Slippers ${ }^{2}$ and Oleg Reva ${ }^{1}$
}

\begin{abstract}
Background: Genomic islands (Gls) are inserts of foreign DNA that have potentially arisen through horizontal gene transfer (HGT). There are evidences that Gls can contribute significantly to the evolution of prokaryotes. The acquisition of Gls through HGT in eukaryotes has, however, been largely unexplored. In this study, the previously developed GI prediction tool, SeqWord Gene Island Sniffer (SWGIS), is modified to predict Gls in eukaryotic chromosomes. Artificial simulations are used to estimate ratios of predicting false positive and false negative Gls by inserting Gls into different test chromosomes and performing the SWGIS v2.0 algorithm. Using SWGIS v2.0, Gls are then identified in 36 fungal, 22 protozoan and 8 invertebrate genomes.

Results: SWGIS v2.0 predicts Gls in large eukaryotic chromosomes based on the atypical nucleotide composition of these regions. Averages for predicting false negative and false positive Gls were $20.1 \%$ and $11.01 \%$ respectively. A total of 10,550 Gls were identified in 66 eukaryotic species with 5299 of these Gls coding for at least one functional protein. The EuGl web-resource, freely accessible at http://eugi.bi.up.ac.za, was developed that allows browsing the database created from identified Gls and genes within Gls through an interactive and visual interface.
\end{abstract}

Conclusions: SWGIS v2.0 along with the EuGI database, which houses Gls identified in 66 different eukaryotic species, and the EuGl web-resource, provide the first comprehensive resource for studying HGT in eukaryotes.

Keywords: Genomic island, Horizontal gene transfer, SWGIS v2.0, Eukaryotes, Comparative genomics, Software tools

\section{Background}

Genomic islands (GIs) are genomic fragments that resulted from the insertion of mobile genetic elements and have been shown to facilitate the evolutionary processes in bacteria and archaea $[1,2]$. Horizontal gene transfer (HGT) allows organisms to rapidly adapt to fluctuating environments and different ecological pressures or opportunities [3, 4]. Studies of bacterial pan-genomes suggest that microbial genomes continuously harvest new genetic information through HGT from a pool of genetic material available as "free goods" and that the accessory genome contributes significantly to bacterial niche speciation [5].

\footnotetext{
* Correspondence: edohan.clasen@fabi.up.ac.za

${ }^{1}$ Centre for Bioinformatics and Computational Biology; Department of

Biochemistry, Genetics and Microbiology, University of Pretoria, Pretoria 0002,

Private Bag X20, Hatfield 0028, South Africa

${ }^{2}$ Forestry and Agricultural Biotechnology Institute; Department of

Biochemistry, Genetics and Microbiology, University of Pretoria, Pretoria 0002, South Africa
}

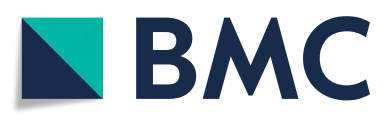

(c) The Author(s). 2018 Open Access This article is distributed under the terms of the Creative Commons Attribution 4.0 International License (http://creativecommons.org/licenses/by/4.0/), which permits unrestricted use, distribution, and

reproduction in any medium, provided you give appropriate credit to the original author(s) and the source, provide a link to the Creative Commons license, and indicate if changes were made. The Creative Commons Public Domain Dedication waiver (http://creativecommons.org/publicdomain/zero/1.0/) applies to the data made available in this article, unless otherwise stated. in the evolution of pathogenesis in virulent microbes [6]

Research of HGT in prokaryotes far exceeds that of eukaryotes. There is, however, compelling examples in some eukaryotic species of where HGT allowed ecological niche speciality $[7,8]$, drove metabolic innovation and expansion $[9,10]$ and accelerated the adaptation to completely novel lifestyles [11, 12]. Albeit less frequent than in prokaryotes, horizontally transferred genes in eukaryotic genomes can clearly also provide an important selective advantage for these species. The impact of HGT on all domains of life are therefore becoming increasingly evident [13].

Methods of GI detection is broadly divided into those using a parametric (or surrogate) or a comparative approach [14]. Parametric methods inherently rely on the comparison of the sequence composition of GIs to that of the surrounding genome or to the average sequence composition of the genome. In contrast, comparative 
methods rely on identification of incongruence in taxonomic relations between organisms and phylogenetic inferences based on selected chromosomal loci [4, 14, 15]. Both parametric and comparative methods have been shown to have advantages and disadvantages. Both produce false positives and false negative errors [16-19], especially between closely related taxa, in weakly sampled taxonomic lineages and for genes that evolve at unusual rates $[14,20]$. Using both approaches to complement each other is therefore preferable.

Studies of HGT in eukaryotes to date focussed on identifying horizontally transferred genes with comparative methods rather than the identification of inserts of GIs with parametric methods. Potentially transferred genes were detected either through exhaustive BLAST searches to identify genes with a higher sequence similarity to bacterial homologs compared to eukaryotic homologs [10, 21], or by phylogenetic incongruence between gene and species trees $[9,22]$. Exploiting the sequence composition bias of GIs with parametric methods commonly used in prokaryotes, is consequently limited in eukaryotes [23, 24]. A possible reason for this is that parametric methods can be computationally intensive on large eukaryotic genomes and that large heterogeneous chromosomes can lead to high rates of false positive identification.

The main objective of this study is to test and modify the algorithm of the prokaryotic GI search tool, SWGIS, as a rapid tool for initial identification of potential GIs in eukaryotic chromosomes. The modified search tool, SWGIS v2.0, is then used to predict GIs in the genomes of a representative sample of fungal, protozoan and invertebrate species in order to determine how common such events are across this range of eukaryotes. Finally, we aim to develop a database and associated search tools of eukaryotic GIs to aid in eukaryotic HGT studies, similar to what exists for prokaryotes.

\section{Implementation}

Identification of genomic islands

SeqWord Gene Island Sniffer (SWGIS) algorithm [19] was modified to SWGIS v2.0 and used for prediction of GI locations in chromosomal sequences. Oligonucleotide usage pattern (OUP) was denoted as a matrix of deviations $\Delta_{[\xi 1 \ldots \xi N]}$ of observed over expected counts of all possible tetranucleotide permutations:

$$
\Delta_{\left[\xi_{1} \ldots \xi_{N}\right]}=\left(C_{\left[\xi_{1} \ldots \xi_{N}\right] \mid o b s}-C_{\left[\xi_{1} \ldots \xi_{N}\right] \mid e}\right) / C_{\left[\xi_{1} \ldots \xi_{N}\right] \mid 0}
$$

where $\xi_{\mathrm{n}}$ is any nucleotide $\mathrm{A}, \mathrm{T}, \mathrm{G}$ or $\mathrm{C}$ in the $N$-long word (in the case of tetranucleotides $N=4$ ); $C_{[\xi 1 \ldots \xi N] \mid o b s}$ is the observed count of the word $\left[\xi_{1} \ldots \xi_{N}\right] ; C_{[\xi 1 \ldots \xi N] \mid e}$ is the expected count and $C_{[\xi 1 \ldots \varepsilon N] \mid 0}$ is a standard count estimated from the assumption of an equal distribution of words in the sequence: $\left(C_{[\xi 1 \ldots \xi N] \mid 0}=L_{\text {seq }} \times 4^{-N}\right)$.
Expected counts of words $C_{[\xi 1 \ldots \xi N] \mid e}$ were calculated in accordance to the applied normalization scheme. Thus, $C_{[\xi 1 \ldots \xi N] \mid e}=C_{[\xi 1 \ldots \xi N] \mid 0}$ if $\mathrm{OU}$ is not normalized, or $C_{[\xi 1 \ldots \xi N] \mid e}=C_{[\xi 1 \ldots \varepsilon N] \mid n}$ if $\mathrm{OU}$ is normalized by empirical frequencies of shorter words of the length $n$ by Markov n-order chain normalization.

Two approaches of normalization by GC content have been exploited where the GC content was calculated either for the sliding window sequence (local normalization) or for the complete reference sequence (generalized normalization).

The distance $(D)$ between two OUPs was calculated as the sum of absolute distances between ranks of identical words ( $w$, in a total $4^{N}$ different words that is 256 for tetranucleotides) after ordering of the words by $\Delta_{[\xi 1 \ldots \xi N]}$ values (eq. 1) in two patterns $i$ and $j$ :

$$
D(\%)=100 \times \frac{\sum_{w}^{4^{N}}\left|\operatorname{rank}_{w, i}-\operatorname{rank}_{w, j}\right|-D_{\min }}{D_{\max -D_{\min }}}
$$

Pattern skew (PS) is a particular case of D where patterns $i$ and $j$ were calculated for the same DNA molecule but for the direct and reversed strands, respectively. $\mathrm{D}_{\max }=4^{N} \times\left(4^{N}-1\right) / 2$ and $\mathrm{D}_{\min }=0$ when calculating a $\mathrm{D}$, or, in the case of PS calculation, $\mathrm{D}_{\min }=4^{N}$ if $N$ is an odd number or $\mathrm{D}_{\min }=4^{N}-2^{N}$ if $N$ is an even number.

Variance of an OU pattern was calculated by the following equation:

$$
V=\frac{\sum_{w}^{4^{N}} \Delta_{w}^{2}}{\left(4^{N}-1\right) \times \sigma_{0}}
$$

where $\mathrm{N}$ is the word length; $\Delta^{2}{ }_{w}$ is the square of a word $w$ count deviation (eq. 1); and $\sigma_{0}$ is the expected standard deviation:

$$
\sigma_{0}=\sqrt{0.02+\frac{4^{N}}{L_{\text {seq }}}}
$$

where $L_{\text {seq }}$ is the sequence length, and $\mathrm{N}$ is the word length.

SWGIS v2.0 calculates two types of variances for the patterns normalized by the GC content of a sliding window (relative variance or RV) and normalized by the GC content of the whole reference sequence (generalized relative variance or GRV). The ratio RV/GRV is then used for GI prediction. These parameters were described in more detail in previous publications [25, 26], where cut-off values for GI predictions were established empirically as the following: D larger than 1.5, PS smaller than 55 and RV/GRV larger than 1.5. 
The principle improvement in SWGIS v2.0 was in calculating a reference OUP for a $300 \mathrm{kbp}$ sliding window and recalculating $\mathrm{OU}$ for every $100 \mathrm{kbp}$. The original SWGIS algorithm calculated a reference OUP for an entire bacterial chromosome which is not representative of more heterogeneous chromosomal fragments in larger eukaryotic chromosomes. Also, in SWGIS v2.0, operons of genes encoding ribosomal RNA ( $r r n$ ) were filtered out by high PS values as well as BLASTN against the SILVA database of $r r n$ sequences of both eukaryotes and prokaryotes [27].

\section{Test chromosomes used for artificial insertions of genomic islands}

To estimate rates of false positive and false negative GI predictions, chromosomes with artificial GI insertions were created. These test chromosomes were chosen based on a preliminary run of SWGIS v2.0 to identify chromosomes that are naïve (those with no predicted GIs) and those that are non-naive (containing other predicted GIs). From there, the relevant chromosomes were chosen to adequately represent the different kingdoms available in the database. The following naïve chromosomes were used: Candida albicans (NW_139454, NW_ 139474), Thalassiosira pseudonana (NC_012068, NC_ 012069), Torulaspora delbrueckii (NC_016501, NC 016504), Phaeodactylum tricornutum (NC_011690, NC_ 011693). The following non-naïve chromosomes were used: Aspergillus fumigatus (NC_007194, NC_007194), Fusarium oxysporum (CM000593, CM000594), Saccharomyces cerevisiae (BK006941, BK006942), Cryptococcus neoformans (NC_026749, NC_026750), Theileria parva (NC_007344, NC_007345), Plasmodium falciparum (NC_004329, NC_004330), Drosophila melanogaster (NC_004353, NC_00454), Caenorhabditis elegans (NC_ 003279, NC_003280).

\section{False negative estimation of SWGIS v2.0}

The Pre_GI [28] database were inspected for GIs that are also contained within the pathogenicity islands database (PAIDB) [29]. Of these, GIs that contained rrn sequences were discarded which related to a total of 194 GIs. The sequences of these 194 GIs were inserted into arbitrary locations of different test chromosomes using a randomization simulation. Each simulation inserted a single GI into an arbitrary location, implemented the SWGIS v2.0 algorithm and determined whether the algorithm identified the artificially inserted PAIDB GI. Thus, on each naïve or non-naïve test chromosome, 194 simulations were performed; each simulation with a different PAIDB GI, and a false negative ratio was determined based on the frequency of correctly detecting the inserted PAIDB GI and incorrectly not detecting the inserted PAIDB GI for each test chromosome.

\section{False positive estimation of SWGIS v2.0}

Random genomic fragments, acting as artificial GIs, were arbitrarily transferred between two chromosomes of the same organism with a randomization simulation. The assumption was made that the OU of two chromosomes of the same organism would be similar and detecting an artificially transferred segment can be considered as a false positive. Each simulation transferred a single arbitrary genomic fragment of $28,173 \mathrm{bp}$, the average length of the 194 GIs from PAIDB used for false negative estimation, from one chromosome to an arbitrary location on the other chromosome, implemented the SWGIS v2.0 algorithm and determined whether the artificially transferred segment was identified as a GI. Specifically, for non-naive chromosomes, the simulation ensured that the transferred fragments do not already contain a GI(s) and is not inserted in locations that already contain a GI(s). For each test chromosome, a total of 100 simulations were performed; each simulation with a single arbitrary genomic fragment inserted into an arbitrary location and a false positive ratio was determined based on the frequency of correctly not detecting the transferred fragment and incorrectly detecting the transferred fragment.

\section{Case studies}

SWGIS v2.0 was used to identify GIs in the genomes of Aspergillus fumigatus and Drosophila ananassae as two case studies. Firstly, we compared GIs identified by SWGIS v2.0 in $A$. fumigatus to previously predicted atypical regions in this organism where the variation in OUP across the genome was also used to predict GIs [23]. In the cited work, GI identification was performed by using a parametric method based on local variations of genomic signatures that makes this study useful for benchmarking of SWGIS v2.0. Secondly, we tested for sequence similarity with BLASTN (e-value cut off $1^{-20}$ ) between GIs predicted in $D$. ananassae to the Wolbachia endosymbiont (NZ_ AAGB00000000) of this species, as previous reports have shown that the entire genome of Wolbachia has been transferred to the D. ananassae genome [30].

\section{Genome sequences for database construction}

Complete sequences of 1062 chromosomes of 66 eukaryotic organisms were obtained from the RefSeq database in GenBank format using the NCBI FTP server (ftp://ftp.ncbi.nih.gov/genomes/refseq). The RefSeq database was chosen to ensure only high quality assemblies in chromosome format was used and to limit the identification of potential bacterial contaminants, especially in smaller contigs of incompletely sequenced genomes. The EuGI web-resource contains the genome accession numbers of all the sequences used (http://eugi.bi.up.ac.za/ eugi_source.php). 


\section{Database software and programming}

MySQL package v5.1.73 for Linux was used for database creation. All programming was performed in Python 2.5.

\section{Results}

Prediction of genomic islands in eukaryotic chromosomes using SWGIS v2.0

The basic principle behind the SWGIS algorithm consisted in a superimposition of values of several statistical parameters calculated for a sliding window. Particularly, GIs were characterized by an increased distance between local tetranucleotide usage patterns and the reference pattern calculated for the complete genome (D-values) and by increased ratio of locally normalized over globally normalized values of local tetranucleotide usage variances (RV/GRV). The approach proved to be useful for distinguishing between horizontally acquired inserts of GI and the core genome sequence, but also this technique allowed discrimination of GIs from other atypical regions such as multiple repeats or loci with an alternative GC content.

Application of the initial version SWGIS v1.0 designed for prokaryotic organisms cased sometimes an increased false prediction of GIs in eukaryotes. Eukaryotic chromosomes are larger and have a higher intra-sequence heterogeneity compared to prokaryotes and in many cases the OUP calculated for large parts of the same chromosome may differ significantly from local OUP. Calculating a reference OUP of $300 \mathrm{kbp}$ and recalculating OU for every step of $100 \mathrm{kbp}$ in SWGIS 2.0 allowed DNA homogeneity within the reference sliding window and can more accurately predict regions that deviate in its oligonucleotide composition compared to the OUP of the rest of the chromosome. This reduced potential false positive prediction. Another improvement in SWGIS v2. 0 was the visual representation of GIs on large linear chromosomes in the form of a scalable vector graphic (Fig. 1). Average computational time for all test chromosomes was approximately $23.66 \mathrm{~s} / \mathrm{kb}$ on a normal Windows desktop.

\section{False negative estimation of SWGIS v2.0}

Calculating false negative values as the ratio of identifying an artificially inserted GI versus not identifying the imitation GI, showed low values in general. The highest false negative percentage was $42.78 \%$ and the lowest was $5.67 \%$. Although a wide range of false negative percentages was observed, the means were $20.8 \%$ and $19.4 \%$ for naïve (Table 1) and non-naïve (Table 2) chromosomes respectively, with a combined average of $20.1 \%$.

\section{False positive estimation of SWGIS v2.0}

Calculating false positive values as the ratio of identifying an artificial inter-chromosomal transfer versus not identifying the insert were low in general except for chromosome 4 of D. melanogaster (NC_004353; 49\%). False positive values showed a wide range for both naïve (Table 1) and non-naïve (Table 2) chromosomes, with means of $3.88 \%$ and $18.13 \%$, respectively, and a combined average of $11.01 \%$. Several naïve sequences had false positives values of $0 \%$.

\section{Case studies \\ Aspergillus fumigatus}

SWGIS v2.0 identified 141 GIs in the genome of $A$. fumigatus. Previously, 189 atypical regions were identified in this genome (Table 3) [23]. However, several of

Aspergillus fumigatus Af293 chromosome 1, whole genome shotgun

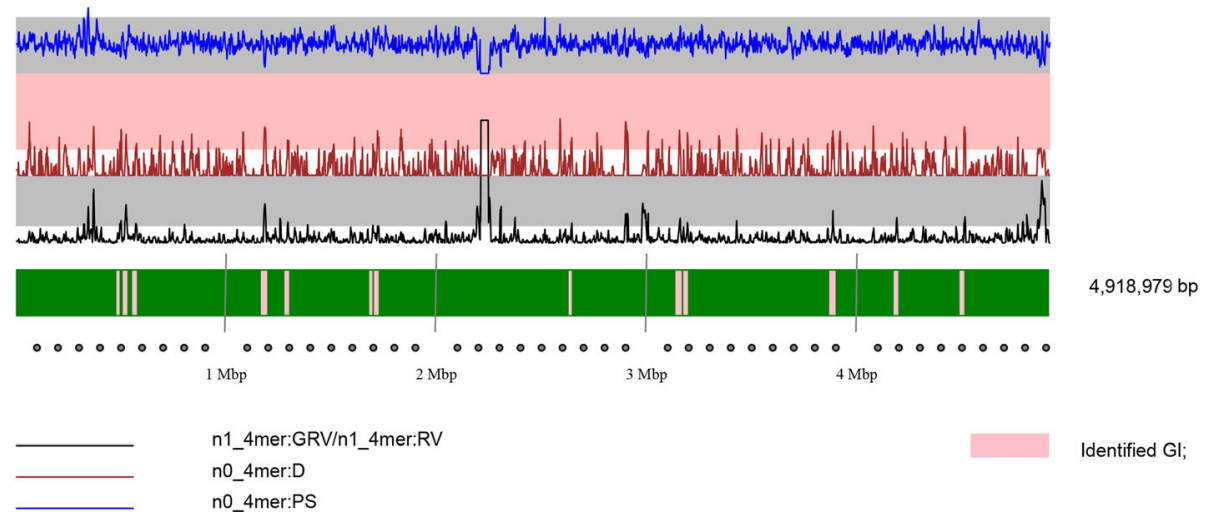

Fig. 1 Graphical output of the SWGIS v2.0 algorithm. The SWGIS v2.0 displays GIs (pink blocks) on large linear chromosomes. Several parameters are also shown on the graph: GC-content (black curve); ratio of generalized to local relative variances calculated for tetranucleotide usage patterns normalized by the GC-content (blue curve, n1_4mer:GRV/n1_4mer:RV); distances between not-normalized local tetranucleotide usage pattern and the global pattern calculated for the complete chromosome (red curve, n0_4mer:D); asymmetry between not-normalized tetranucleotide usage patterns calculated for the direct and complement DNA strands (green curve, n0_4mer:PS). Use of these parameters for GI identification and their standard abbreviations were explained in more detail by Ganesan et al. (2008). and on the EuGl website (http://eugi.bi.up.ac.za) [25] 
Table 1 False positive and false negative values of SWGIS v2.0 calculated for different naïve chromosomes

\begin{tabular}{|c|c|c|}
\hline & False positive $^{a}$ & False negative \\
\hline C. albicans NW_139454 & $16 \%$ & $5.67 \%$ \\
\hline C. albicans NW_139474 & $0 \%$ & $7.73 \%$ \\
\hline T. pseudonana NC_012068 & $0 \%$ & $30.93 \%$ \\
\hline T. pseudonana NC_012069 & $0 \%$ & $30.41 \%$ \\
\hline T. delbrueckii NC_016501 & $1 \%$ & $16.49 \%$ \\
\hline T. delbrueckii NC_016504 & $2 \%$ & $21.13 \%$ \\
\hline P. tricornutum NC_011690 & $11 \%$ & $24.74 \%$ \\
\hline P. tricornutum NC_011693 & $1 \%$ & $29.90 \%$ \\
\hline
\end{tabular}

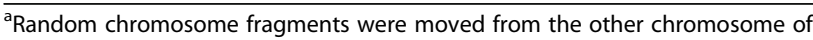
the same organism (e.g. for NW_139454 random fragments were moved from NW_139474 to NW_139454 and vice versa for NW_139474)

the previously identified GIs were either small $(<5 \mathrm{~kb})$ or contained $r r n$ sequences (Fig. 2). After filtering out small and $r r n$ containing GIs from the 189 previously identified GIs (filtering function is embedded in the SWGIS v2.0 algorithm), a subset of only 31 GIs remained for comparison. From this smaller subset of 31 GIs, a total of 18 GIs were confirmed by SWGIS v2.0. Of the remaining 13 GIs of the smaller subset not predicted as GIs by SWGIS v2.0, only five contained MGEs according to the definition used in the original publication [23] and thus represent true positives not identified by SWGIS v2.0. It can therefore be argued that only five GIs were missed by SWGIS v2.0 as false negatives.

\section{Drosophila ananassae}

SWGIS v2.0 identified 1288 GIs in the genome of $D$. ananassae (using only scaffolds larger than $300 \mathrm{kbp}$ ). Of these, 70 were coding GIs and 1218 were non-coding GIs.

Table 2 False positive and false negative values of SWGIS v2.0 calculated for different non-naïve chromosomes

\begin{tabular}{lll}
\hline & False positive $^{a}$ & False negative \\
\hline Fungi & & \\
A. fumigatus NC_007194 & $18 \%$ & $30.93 \%$ \\
F. oxysporum CM000593 & $16 \%$ & $34.54 \%$ \\
S. cerevisisae BK006941 & $2 \%$ & $11.34 \%$ \\
C. neoformans NC_026749 & $7 \%$ & $42.78 \%$ \\
Protozoa & & \\
T. parva ${ }^{\text {NC_007344 }}$ & $25 \%$ & $7.22 \%$ \\
P. falciparum NC_004329 & $22 \%$ & $5.67 \%$ \\
Invertebrates & & \\
D. melanogaster NC_004353 & $49 \%$ & $13.40 \%$ \\
C. elegans & & \\
\hline
\end{tabular}

${ }^{a}$ Random chromosome fragments were moved from the successive chromosome of the one listed in the table (e.g. for A. fumigatus random fragments were moved from NC_007195 to NC_007194, and similarly for the other organisms listed in column one)
Table 3 Distribution of Gls identified by SWGIS v2.0 versus Mallet et al.

\begin{tabular}{llllllllll}
\hline Chromosome \# & 1 & 2 & 3 & 4 & 5 & 6 & 7 & 8 & Total \\
\hline \#Gls - Mallet et al. & 30 & 28 & 28 & 22 & 22 & 31 & 14 & 14 & 189 \\
\#GIS - SWGIS v2.0 & 19 & 24 & 26 & 17 & 16 & 20 & 11 & 8 & 141 \\
\hline
\end{tabular}

BLASTN alignment showed a very high sequence similarity between coding GIs and regions in the genome of the Wolbachia endosymbiont of $D$. ananassae (Fig. 3). In some instances, an entire contig of Wolbachia showed high sequence similarity to GIs of $D$. ananassae, for example contig NZ_AAGB01000133.1 had an average identity of $93.48 \%$ against GIs of $D$. ananassae. Furthermore, many GIs identified in D. ananassae constitute fragments of the genome of Wolbachia endosymbiont NZ_AAGB00000000.1.

\section{EuGI database development and analysis}

Using SWGIS v2.0 a total of 10,550 GIs were identified in 66 eukaryotic species (Additional file 1: Table S1). All species analysed contained at least one GI, however, 176 fungal chromosomes, 34 protozoan chromosomes and 11 invertebrate chromosomes were naïve, i.e. do not contain any GIs. An overview of GI prediction in different genomes is shown in Table 4.

Identified GIs were subsequently filtered for those that are coding i.e. contained at least one protein coding gene within the GI and those that are non-coding i.e. contained no protein coding genes. Coding GIs were 5299 in total. Large invertebrate genomes contained a large proportion of non-coding GIs (Table 4).

Comparison of Gl sizes identified by SWGIS v2.0 versus Mallet et al.

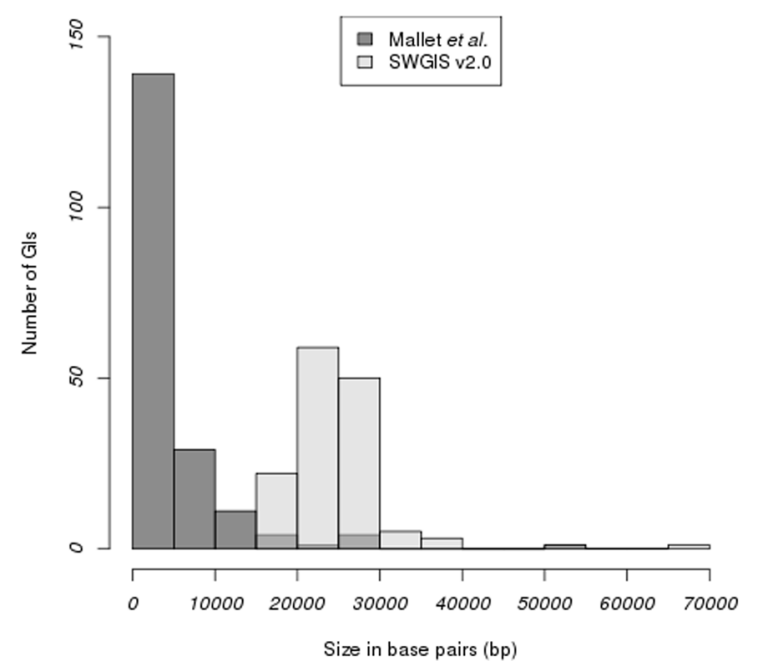

Fig. 2 Comparison of Gl sizes identified by Mallet et al. and SWGIS V2.0 


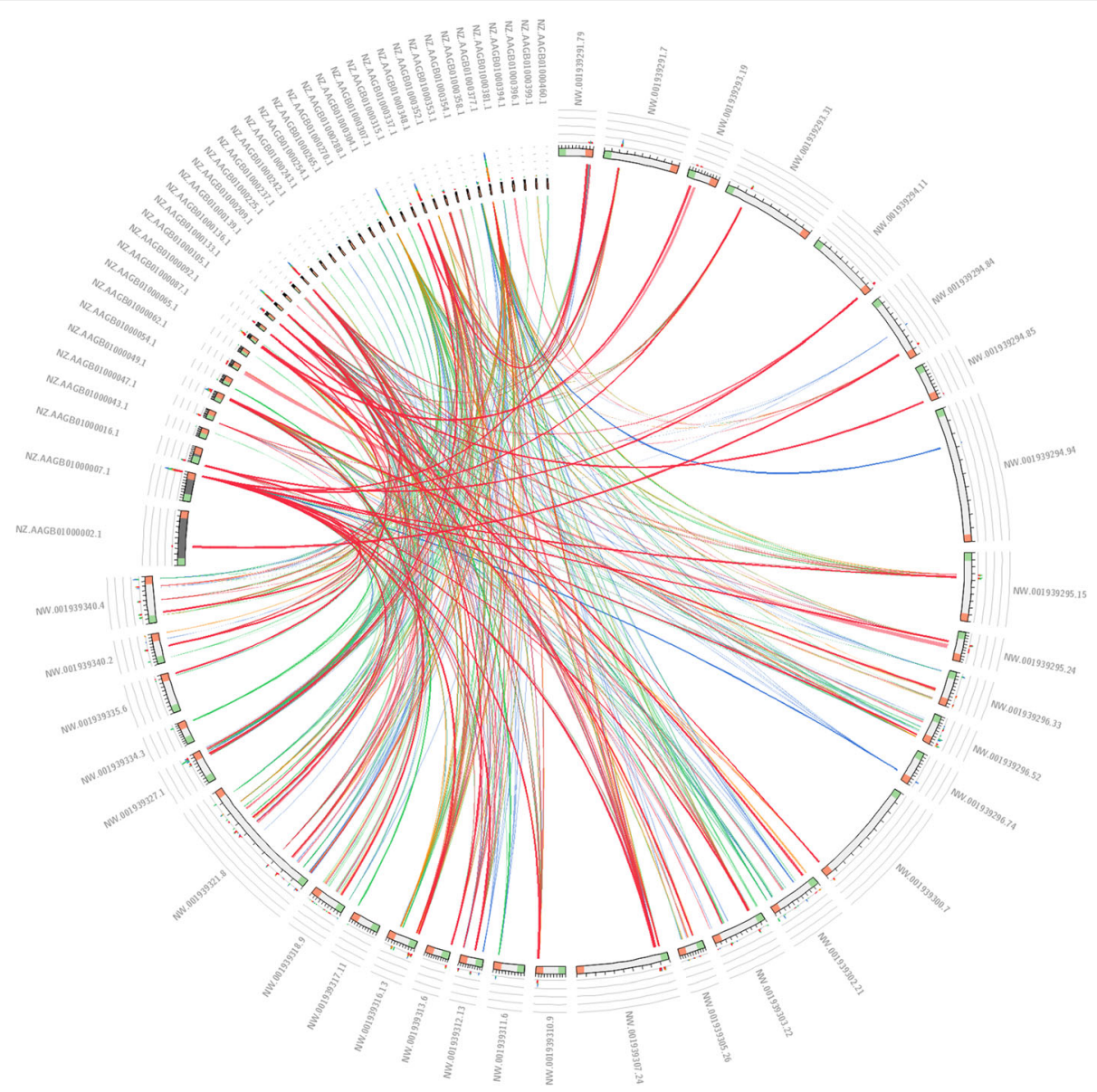

Fig. 3 BLASTN alignment of coding Gls identified in D. ananassae and the Wolbachia endosymbiont of D. ananassae. Circoletto [39] was used to visualize sequence similarity of coding Gls of D. ananassae and its Wolbachia endosymbiont. Scaffolds of Wolbachia are represented by their accession numbers from the original GenBank file downloaded from NCBI. Predicted Gls from D. ananassae are indicated by the scaffold accession number (e.g. NW.001939327) followed by a number which indicate the number of the Gl predicted in the specific scaffold (e.g. NW. 001939327.1). Line colours are indicative of e-values between queries and subjects with red the smallest, orange second, green third and blue the highest e-values

To avoid enriching the database with non-coding elements abundant in eukaryotic genomes, only coding GIs and 28,943 potentially horizontally transferred genes comprising coding GIs were used for further comparative analyses. BLASTN and BLASTP (e-value cut-off 0. 01) of 1472 GI nucleotide sequences and 9831 protein translations of constituent genes showed a significant level of sequence similarity against archaeal/bacterial GIs

Table 4 Gls predicted in different eukaryotic lineages using SWGIS V2.0

\begin{tabular}{lllll}
\hline & \# species & \# chromosomes & \# Gls & \# coding Gls \\
\hline Fungi & 36 & 614 & 3080 & 2299 \\
Protozoa & 22 & 392 & 2911 & 2506 \\
Invertebrate & 8 & 56 & 4559 & 494 \\
Total & 66 & 1062 & 10,550 & 5299 \\
\hline
\end{tabular}

residing in the Pre_GI database [28]. GI sequences, constituent genes, which potentially have been acquired by HGT, and the results of BLAST searches against the Pre_GI database were then used to construct the Eukaryotic Genomic Islands (EuGI) database. The database enables comparison of novel predicted GIs in user data against GIs already predicted in 66 eukaryotic species and stored in the database.

A variety of functions were associated with predicted genes of HGT origin. A total of 17,715 genes were either hypothetical or uncharacterized in their annotation. Genes encoding for different tRNA molecules were found in 342 predicted GIs. Other functional classes of GI-associated genes include 406 kinases, 167 transporter proteins, 166 oxidoreductases, 155 phosphatases, 107 peptidases and 77 hydrolases. 


\section{The EuGl web-resource}

To easily visualise and browse eukaryotic GIs predicted by SWGIS v2.0 and the involved genes, the EuGI database was made freely available through the EuGI webresource (http://eugi.bi.up.ac.za). Genomic sequences of GIs in addition to protein sequences of predicted genes can be downloaded through the resource. Different search and browse tools as well as queries with user data are incorporated into the EuGI web-resource.

SWGIS v2.0 was implemented in the web-resource as a eukaryotic GI finder for users to predict GIs in their own data. This tool allows the upload of either genomic sequences in FASTA format or annotated sequences in GenBank format.

\section{Discussion}

In this study, the SWGIS GI predictor was adapted for the use in eukaryotic genomes, termed SWGIS 2.0. The algorithm of SWGIS v2.0 was shown to have acceptable levels of false negative and false positive estimates of $20.1 \%$ and $11.01 \%$, respectively. GIs were predicted using SWGIS v2. 0 in 66 different fungal, protozoan and invertebrate genomes. All predicted GIs and potentially horizontally transferred genes within these GIs, together with a repertoire of tools for GI identification are also combined in the EuGI database and web-resource (http://eugi.bi.up.ac. za). This provides one of the first comprehensive analytical suites for GI investigation in eukaryotes.

SWGIS v2.0 is one of the first parametric GI predictors for eukaryotes, and the first that has been tested on a broad range of taxa. While several programs exist that apply parametric algorithms on prokaryotic genomes to identify GIs $[1,2,31]$, the identification of GIs in eukaryotic genomes are limited to a single parametric GI predictor optimized for eukaryotes [32] and GI studies of A. fumigatus [23]. These two studies were applied to smaller subsets of species or focussed on a single species [23, 32]. Furthermore, rates of false positive and false negative GI predictions in eukaryotic genomes were also estimated in this study, but this information is not available for other parametric GI predictors designed for eukaryotes.

A reference OUP is calculated in the SWGIS v2.0 algorithm for a large $(300 \mathrm{kbp})$ sliding window of the chromosome and recalculated for each step of $100 \mathrm{kbp}$. OUPs have been shown to be constant genomic signatures and characteristic for whole bacterial chromosomes except for HGT acquisitions, clusters of genes for ribosomal RNA and some other minor loci [26]. However, variations of OUPs across eukaryotic chromosomes have not been explored on a large set of different species, as was done here. The sliding window optimization of the SWGIS program [15] in SWGIS v2.0 accounts for the sequence heterogeneity within eukaryotic chromosomes and allowed the algorithm to distinguish between GIs and other atypical regions.
The rate of false positive predictions in SWGIS v2.0 was reduced by embedded filtering algorithms including filtering of loci containing $r r n$ genes and those smaller than $5 \mathrm{kbp}$. These filters are commonly used to limit false positive prediction in prokaryotic GIs [26, 31]. Accounting for different OUP statistical parameters in SWGIS v2.0 allows distinguishing GIs from multiple other genomic loci with alternative OUPs, i.e. noncoding repeat elements or intergenic regions that reduces false positive predictions.

False positive and false negative values for SWGIS v2.0 are comparable to those from common prokaryotic GI predictors [2]. For example, a comparison between four routinely used prokaryotic GI predictors, SIGI-HMM, PAI-IDA, Centroid and Alien_Hunter (IVOM) showed ranges of $23 \%-72 \%$ and $8 \%-62 \%$ for sensitivity and specificity, respectively [2]. The false negative average calculated for SWGIS v2.0 (20.1\%) indicates a better sensitivity of the program than those reported for above mentioned GI predictors. The false positive average calculated for SWGIS v2.0 (11\%) is within the specificity range reported for these prokaryotic GI predictors.

The identification of GIs with SWGIS v2.0 can alleviate the task of searching through an entire genome for HGT events by predicting inserts of GIs comprising functional genes. Phylogenetic incongruence between gene and species trees is often considered as the 'golden standard' for confirming HGT in eukaryotes and is more broadly used to investigate HGT in eukaryotes [5, 9]. Relying solely on phylogenetics to identify GIs have also been shown to underestimate the rate of HGT events in eukaryotes and can consequently result in high frequencies of false negatives [19]. The reason for this is that the sensitivity of these methods is limited by availability of genome sequences of putative 'GI donor' organisms in public databases, which won't be fully comprehensive in the near future. Contrary, parametric OUP based methods allow GI prediction without need for any reference genome that is an important concept in prokaryotic HGT studies [2, 15, 31]. Although downstream phylogenetic analyses remain critical, screening a genome using SWGIS v2.0 to identify GIs can provide a focus for further phylogenetic and functional annotation studies.

The web-accessible EuGI database is the first of its kind resource for eukaryotic GIs. Several online databases of GIs in prokaryotes exist [28, 29, 33], but none for eukaryotes. The EuGI web-resource allows easy and user-friendly browsing of GIs currently residing in the EuGI database through the web-interface. The SWGIS v2.0 program forming part of the EuGI web-resource enables users to easily predict GIs without having to download and compile the program code.

A wide range of functions was associated with genes within identified GIs. tRNA genes have been described to act as integration sites for foreign DNA and can be 
used as supporting evidence for GI identification [2, 34, 35]. Identifying a high frequency of tRNA genes associated with GIs predicted by SWGIS v2.0 therefore demonstrates the ability of the program to identify atypical regions in large genomes. Versatile functional classes of genes, such as kinases, oxidoreductases, phosphatases, peptidases and hydrolases, were associated with GIs identified in this study. Several of these functions have been shown in other studies to be associated with HGTs $[10,21,23]$. This suggests that HGT potentially contributed to the evolutionary adaptation of eukaryotic organisms studied here [36]. Comparative and phylogenetic studies of all horizontally acquired genes remains critical to conclude that these are indeed true positive HGT events [2]. The results can, however, now serve as a benchmark for further functional characterization and to study the role of HGT in the genome evolution of different eukaryotic species more extensively.

The SWGIS v2.0 program was applied to the genomes of A. fumigatus and D. ananassae, where HGT have been shown to constitute to both these organisms [23, 30]. Previously predicted GIs in A. fumigatus is the only report for horizontally acquired genomic loci in a eukaryotic organism [23]. We were able to filter out several GIs previously predicted in A. fumigatus and reduce the set used for comparison against GIs predicted by SWGIS v2.0, because previous reports showed that GIs containing rrn genes were most likely false positives and those smaller than $5 \mathrm{kbp}$ were statistically unreliable for the used method [2, 26]. A significant overlap existed between GIs predicted by SWGIS v2.0 and those previously predicted after applying the described filtering. SWGIS v2.0 therefore proved to be more consistent for GI prediction in A. fumigatus [2].

The sequence similarity between GIs predicted by SWGIS v2.0 in D. ananassae and the Wolbachia endosymbiont of this organism confirms the high frequency of DNA exchange between these organisms. This is consistent with previous studies that reported that the entire Wolbachia genome had been horizontally transferred to the nuclear genome of $D$. ananassae [30]. Endosymbiont-to-host HGT can be frequent and is a well-studied class of gene exchange in eukaryotes [30, 37, 38]. The results of this study supports the conclusion that horizontally transferred genomic segments can successfully be identified using a parametric algorithm such as SWGIS v2.0.

\section{Conclusions}

Here we reported one of the first GI predictors for eukaryotes, SWGIS v2.0, which used a modified version of the SWGIS algorithm. Importantly, we reported the first estimates for predicting false positive and false negative GIs for a parametric GI search tool tested on eukaryotes. The results showed that SWGIS v2.0 performed well compared to common prokaryotic GI prediction tools. This tool, along with the EuGI database constructed by applying the SWGIS v2.0 algorithm on 66 different eukaryotic species and implementing it on the EuGI webresource, provide the first comprehensive resource for GI prediction and comparison in higher organisms. Using the SWGIS v2.0 tool along with the EuGI web-resource will greatly aid in studies focussed on analysing the flow of genetic material across taxonomic boundaries. Ultimately, studying of horizontally acquired genes in this manner can contribute to the many unanswered questions about how HGT has influenced the evolution of eukaryotes.

\section{Additional file}

Additional file 1: Table S1. Eukaryotic organisms used for database construction by GI prediction with SWGIS v2.0. The table presents all fungal, protozoan and invertebrate species that were used for Gl prediction in this study along with the amount of Gls predicted in each species and the amount of genes retained within these Gls. (DOCX 17 kb)

\section{Funding}

We thank the National Research Foundation of South Africa for grants 93134 and 93664 to O Reva and grant 102973 to FJ Clasen.

\section{Availability of data and materials}

All data generated or analysed during this study are included in this published article and available at http://eugi.bi.up.ac.za.

Project name: SWGIS v2.0.

Project home page: http://eugi.bi.up.ac.za

Operating system(s): Platform independent.

Programming language: Python 2.5

Other requirements: None.

License: None.

Any restrictions to be used by non-academics: None.

\section{Authors' contributions \\ All authors contributed to conceptualization of the work as well as writing of the manuscript. FJC and REP performed all, including statistical, analyses as well as database and web-development. OR was responsible for development of the SWGIS algorithm. BS contributed to the interpretation of results and editing of the manuscript. All authors read and approved the final manuscript.}

Ethics approval and consent to participate

Not applicable.

Competing interests

The authors declare that they have no competing interests.

\section{Publisher's Note}

Springer Nature remains neutral with regard to jurisdictional claims in published maps and institutional affiliations. 
Received: 23 November 2017 Accepted: 25 April 2018

Published online: 03 May 2018

\section{References}

1. Juhas M, Van Der Meer JR, Gaillard M, Harding RM, Hood DW, Crook DW. Genomic islands: tools of bacterial horizontal gene transfer and evolution. FEMS Microbiol Rev. 2009;33(2):376-93.

2. Langille MGI, Hsiao WWL, Brinkman FSL. Detecting genomic islands using bioinformatics approaches. Nat Rev Microbiol. 2010;8(5):373-82.

3. Boto L. Horizontal gene transfer in evolution: facts and challenges. Proc $R$ Soc London B Biol Sci. 2010;277(1683):819-27.

4. Jain R, Rivera MC, Moore JE, Lake JA. Horizontal gene transfer, genome innovation and evolution. Mol Biol Evol. 2003;20(10):1598-602.

5. Soucy SM, Huang J, Gogarten JP. Horizontal gene transfer: building the web of life. Nat Rev Genet Nature Publishing Group. 2015;16(8):472-82.

6. Hacker J, Blum-Oehler G, Mühldorfer I, Tschäpe H. Pathogenicity islands of virulent bacteria: structure, function and impact on microbial evolution. Mol Microbiol. 1997;23(6):1089-97.

7. Acuña R, Padilla BE, Flórez-Ramos CP, Rubio JD, Herrera JC, Benavides P, et al. Adaptive horizontal transfer of a bacterial gene to an invasive insect pest of coffee. Proc Natl Acad Sci. 2012;109(11):4197-202.

8. Christin PA, Edwards EJ, Besnard G, Boxall SF, Gregory R, Kellogg EA, et al. Adaptive evolution of $C 4$ photosynthesis through recurrent lateral gene transfer. Curr Biol Elsevier Ltd. 2012;22(5):445-9.

9. Schönknecht G, Chen W-H, Ternes CM, Barbier GG, Shrestha RP, Stanke M, et al. Gene transfer from bacteria and archaea facilitated evolution of an extremophilic eukaryote. Science. 2013;339(6124):1207-10.

10. Boschetti C, Carr A, Crisp A, Eyres I, Wang-Koh Y, Lubzens E, et al. Biochemical diversification through foreign gene expression in bdelloid rotifers. PLoS Genet. 2012;8(11):e1003035.

11. Paganini J, Campan-Fournier A, Da Rocha M, Gouret P, Pontarotti P, Wajnberg $E$, et al. Contribution of lateral gene transfers to the genome composition and parasitic ability of root-knot nematodes. PLoS One. 2012; 7(11):e50875.

12. Blaxter ML, Koutsovoulos G. The evolution of parasitism in Nematoda. Parasitology. 2015;142(S1):S26-39.

13. Keeling PJ. Functional and ecological impacts of horizontal gene transfer in eukaryotes. Curr Opin Genet Dev. 2009;19(6):613-9.

14. Ravenhall M, Škunca N, Lassalle F, Dessimoz C. Inferring horizontal gene transfer. PLoS Comput Biol. 2015;11(5):1-16.

15. Bezuidt O, Lima-Mendez G, Reva ON. SeqWord Gene Island sniffer: a program to study the lateral genetic exchange among bacteria. World Acad Sci Eng Technol. 2009;34(58):1169-11274.

16. Ragan MA. On surrogate methods for detecting lateral gene transfer. FEMS Microbiol Lett. 2001;201(2):187-91.

17. Ragan MA, Harlow TJ, Beiko RG. Do different surrogate methods detect lateral genetic transfer events of different relative ages? Trends Microbiol. 2005;14(1):4-8

18. Koski LB, Morton RA, Golding GB. Codon bias and base composition are poor indicators of horizontally transferred genes. Mol Biol Evol. 2001;18(3): 404-12.

19. Andersson JO. Phylogenomic approaches underestimate eukaryotic gene transfer. Mob Genet Elements. 2012;2(1):59-62.

20. Kurland CG, Canback B, Berg OG. Horizontal gene transfer: a critical view. Proc Natl Acad Sci. 2003;100(17):9658-62.

21. Crisp A, Boschetti C, Perry M, Tunnacliffe A, Micklem G. Expression of multiple horizontally acquired genes is a hallmark of both vertebrate and invertebrate genomes. Genome Biol. 2015;16(1):50.

22. Andersson JO, Hirt RP, Foster PG, Roger AJ. Evolution of four gene families with patchy phylogenetic distributions: influx of genes into protist genomes. BMC Evol Biol. 2006;6(1):27.

23. Mallet LV, Becq J, Deschavanne P. Whole genome evaluation of horizontal transfers in the pathogenic fungus aspergillus fumigatus. BMC Genomics. 2010;11(1):171.

24. Fedorova ND, Khaldi N, Joardar VS, Maiti R, Amedeo P, Anderson MJ, et al. Genomic islands in the pathogenic filamentous fungus Aspergillus fumigatus. PLoS Genet. 2008;4(4):e1000046.

25. Ganesan H, Rakitianskaia AS, Davenport CF, Tümmler B, Reva ON. The SeqWord genome browser: an online tool for the identification and visualization of atypical regions of bacterial genomes through oligonucleotide usage. BMC Bioinformatics. 2008;9(1):333.
26. Reva ON, Tümmler B. Differentiation of regions with atypical oligonucleotide composition in bacterial genomes. BMC Bioinformatics. 2005;6:251.

27. Quast C, Pruesse E, Yilmaz P, Gerken J, Schweer T, Yarza P, et al. The SILVA ribosomal RNA gene database project: improved data processing and webbased tools. Nucleic Acids Res. 2013;41(D1):590-6.

28. Pierneef R, Cronje L, Bezuidt O, Reva ON. Pre-Gl: a global map of ontological links between horizontally transferred genomic islands in bacterial and archaeal genomes. Database. 2015;2015(3):1-13.

29. Yoon SH, Park YK, Lee S, Choi D, Oh TK, Hur CG, et al. Towards pathogenomics: a web-based resource for pathogenicity islands. Nucleic Acids Res. 2007;35(suppl_1):D395-400.

30. Hotopp JCD, Clark ME, Oliveira DCSG, Foster JM, Fischer P, Torres MCM, et al. Widespread lateral gene transfer from intracellular bacteria to multicellular eukaryotes. Science. 2007;317(5845):1753-6.

31. Dufraigne C, Fertil B, Lespinats S, Giron A, Deschavanne P. Detection and characterization of horizontal transfers in prokaryotes using genomic signature. Nucleic Acids Res. 2005;33(1):e6.

32. Jaron KS, Moravec JC, Martínková N. SigHunt: horizontal gene transfer finder optimized for eukaryotic genomes. Bioinformatics. 2014;30(8):1-6.

33. Langille MGl, Brinkman FSL. IslandViewer: an integrated interface for computational identification and visualization of genomic islands. Bioinformatics. 2009:25(5):664-5.

34. Hacker J, Kaper JB. Pathogenicity islands and the evolution of microbes. Annu Rev Microbiol. 2000;54(1):641-79.

35. Ou HY, Chen LL, Lonnen J, Chaudhuri RR, Thani A. Bin, smith R, et al. a novel strategy for the identification of genomic islands by comparative analysis of the contents and contexts of tRNA sites in closely related bacteria. Nucleic Acids Res. 2006:34(1):1-11.

36. Shapiro BJ, Leducq JB, Mallet J. What is speciation? PLoS Genet. 2016;12(3): e1005860.

37. Hotopp JCD. Horizontal gene transfer between bacteria and animals. Trends Genet Elsevier Ltd. 2011;27(4):157-63.

38. Nikoh N, Tanaka K, Shibata F, Kondo N, Hizume M, Shimada M, et al. Wolbachia genome integrated in an insect chromosome: evolution and fate of laterally transferred endosymbiont genes. Genome Res. 2008;18(2):272-80.

39. Darzentas N. Circoletto: visualizing sequence similarity with Circos. Bioinformatics. 2010:26(20):2620-1.

\section{Ready to submit your research? Choose BMC and benefit from:}

- fast, convenient online submission

- thorough peer review by experienced researchers in your field

- rapid publication on acceptance

- support for research data, including large and complex data types

- gold Open Access which fosters wider collaboration and increased citations

- maximum visibility for your research: over $100 \mathrm{M}$ website views per year

At BMC, research is always in progress.

Learn more biomedcentral.com/submissions 